\title{
Combined hyperdense gallbladder wall-lumen sign: new computed tomography sign in acute gangrenous cholecystitis
}

\author{
Binit Sureka ${ }^{1 A, B, D, E, F}$, Satya Jha ${ }^{1 B, D}$, Mahaveer S. Rodha ${ }^{2 B, F}$, Ramkaran Chaudhary ${ }^{2 B, F}$, Poonam Elhence ${ }^{3 B, D}$, \\ Pushpinder S. Khera ${ }^{1 B, D}$, Pawan K. Garg ${ }^{1 B, D}$, Taruna Yadav ${ }^{1 B, D}$, Akhil Goel ${ }^{4 C}$ \\ 'Department of Diagnostic and Interventional Radiology, All India Institute of Medical Sciences, Jodhpur, Rajasthan, India \\ 2Department of General Surgery, All India Institute of Medical Sciences, Jodhpur, Rajasthan, India \\ ${ }^{3}$ Department of Pathology and Lab Medicine, All India Institute of Medical Sciences, Jodhpur, Rajasthan, India \\ ${ }^{4}$ Department of Community Medicine, All India Institute of Medical Sciences (AlIMS), Jodhpur, Rajasthan
}

\section{Abstract}

\begin{abstract}
Purpose: The objective of our study was to evaluate the combined hyperdense gallbladder wall-lumen sign on computed tomography (CT) in diagnosing gangrenous cholecystitis.

Material and methods: We retrospectively reviewed the unenhanced CT scans of surgically proven cases of acute gangrenous (GCh) and non-gangrenous cholecystitis (nonGCh). Eleven cases of pathologically proven acute gangrenous cholecystitis and 12 consecutive cases of surgically proven acute non-gangrenous cholecystitis that underwent CT at our institute were included in the study so as to have $1: 1$ control. The Hounsfield unit (HU) value of the gallbladder wall and intraluminal bile was measured. Interobserver variability for individual CT findings was also assessed.

Results: The gangrenous cholecystitis group had significantly higher HU values of wall and bile (median value of $33 \mathrm{HU}$ vs. $21 \mathrm{HU}$ and median value of $21 \mathrm{HU}$ vs. $8.5 \mathrm{HU}$, respectively, $p<0.05$ ). The area under the receiver operator characteristic curve for HU lumen was 0.80 (95\% CI: $0.62-0.98, p=0.014)$ with an ideal cut-off at $31.5 \mathrm{HU}$, where the sensitivity was $54.5 \%$ and specificity was $91.7 \%$. HU lumen has an even better assessment for gangrenous cholecystitis with AUC of its ROC as 0.92 (95\% CI: 0.80-1.00, $p=0.001$ ) with an ideal cut-off at $12.5 \mathrm{HU}$, where the sensitivity was $81.8 \%$ and specificity was $91.7 \%$. The combined wall-lumen cut-off is $35 \mathrm{HU}$ with sensitivity of $100 \%$ and specificity of $75 \%$.
\end{abstract}

Conclusion: A cut-off CT density value of the gallbladder wall of more than $31.5 \mathrm{HU}$, intraluminal bile more than $12.5 \mathrm{HU}$, and combined wall-lumen HU of more than 35 can predict GCh.

Key words: CT, gallbladder, hyperdense, lumen, wall.

\section{Introduction}

Gangrenous cholecystitis (GCh) is a complicated variant of acute cholecystitis due to progressive and profound vascular insufficiency, which results in necrosis and perforation of the gallbladder wall [1]. The mortality rate in GCh is higher, ranging between $15 \%$ and $50 \%$. Due to this increased risk of mortality, these patients often require emergency cholecystectomy [2]. Thus, it is important for a radiologist to diagnose this entity and differentiate it from uncomplicated acute or chronic cholecystitis. Among the various imaging modalities available, ultrasound is the first modality of choice because it is a noninvasive, cost-effective technique that does not involve radiation exposure or iodinated contrast administration [3]. However, computed tomography (CT) may be required in

Correspondence address:

Binit Sureka, Associate Professor, Diagnostic and Interventional Radiology, All India Institute of Medical Sciences, Jodhpur, Basni Industrial Area,

342005, Jodhpur, India, e-mail: binitsurekapgi@gmail.com

Authors' contribution:

A Study design · B Data collection · C Statistical analysis · D Data interpretation · E Manuscript preparation · F Literature search · G Funds collection 
certain cases when the clinical presentation is atypical and suspicious of complication or diagnostic dilemma.

Various CT findings and signs of acute gangrenous cholecystitis have been described in previously published literature. Hyperdense gallbladder wall sign was described in acute gangrenous cholecystitis by Cheng et al. in 2004, who found this sign in $51 \%$ of their cases [4]. However, in a recently published series by Chang et al. [5] they did not find hyperdense wall sign to be a reliable predictor of gallbladder wall ischaemia. There is an overlap between various CT findings to differentiate gangrenous and nongangrenous cholecystitis. To our knowledge, no prior study has been conducted to look both into CT Hounsfield units (HU) of gallbladder wall and intraluminal bile to predict gangrenous change in acute cholecystitis.

The purpose of the study was to review unenhanced CT scans of cases with histopathologically confirmed acute gangrenous cholecystitis and acute non-gangrenous cholecystitis. Our hypothesis was that the density (CT $\mathrm{HU}$ ) of both gallbladder wall and bile increased in cases of acute gangrenous cholecystitis due to early ischaemia, mucosal necrosis, and haemorrhage within the wall and anaerobic bacteria proliferation, suppurative infection, and purulent material filling the gallbladder lumen [6]. To test our hypothesis, we reviewed the density of the gallbladder wall and intraluminal bile in cases of acute gangrenous and non-gangrenous cholecystitis.

\section{Material and methods}

\section{Study population}

We retrospectively reviewed the unenhanced CT scans of surgically proven cases of acute gangrenous and nongangrenous cholecystitis that underwent imaging at our Institute between January 2017 to September 2019. Institutional review board approval was obtained as per our institution's policy. The study population was divided into two groups - acute gangrenous and non-gangrenous cholecystitis. CT scans of 23 patients with acute cholecystitis,

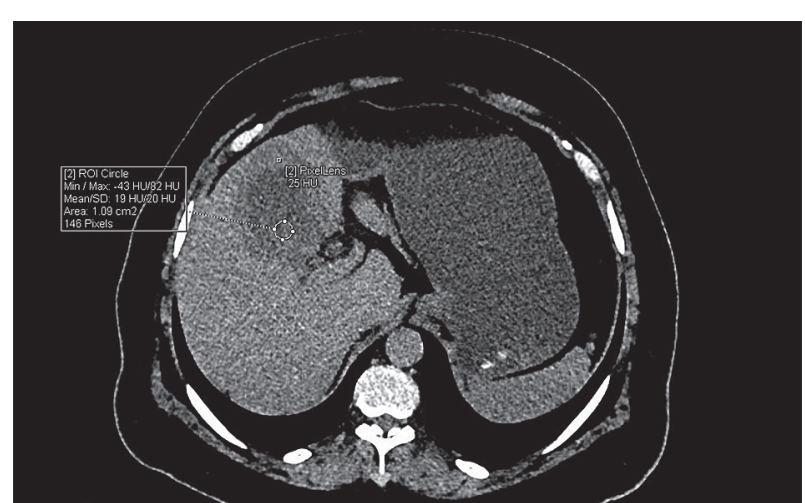

Figure 1. Non-contrast computed tomography scan showing a pixel-based method to calculate gallbladder wall Hounsfield units (HU) and surface area based ROI used to calculate lumen $\mathrm{HU}$ with pathologically proven acute gangrenous cholecystitis in 11 patients and surgically proven acute non-gangrenous cholecystitis in 12 , were included in the study.

\section{Computed tomography technique}

CT abdomen was performed on a Siemens Somatom Definition Flash $2 \times 128$ Dual-source CT scanner (Siemens, Erlangen, Germany). Unenhanced CT examination of the patients was acquired using the following parameters ( $0.5 \mathrm{~s}$ rotation time, pitch value of $0.6,0.75 \mathrm{~mm}$ section thickness, $3 \mathrm{~mm}$ reconstruction interval, $100-120 \mathrm{kVp}$ with Care Dose reference mAs 290). Additional images were reconstructed with $0.5 \mathrm{~mm}$ reconstruction intervals for detailed interpretation.

\section{Image interpretation}

Unenhanced CT scans were reviewed by two radiologists with nine years (B.S.) and four years (S.J.) of experience in abdominal imaging. The HU value of the gallbladder wall and intraluminal bile was measured. Three readings were taken each for gallbladder wall (pixel-based) and intraluminal bile (based on surface area), and an average value was recorded (Figure 1). ROIs were kept away from the gallbladder stone after confirming on ultrasound.

\section{Statistical analysis}

Statistical analyses were performed with SPSS for Windows (version 22.0, SPSS, IBM Corp., Armonk, NY), and differences were considered significant when $p<0.05$. Results were expressed as the median value for continuous variables, absolute frequencies, and percentage for categoric variables. If a variable was not normally distributed, we used the Mann-Whitney $U$ test (Wilcoxon rank-sum test) to obtain the $p$-value. With histologic diagnosis as the reference standard, we calculated sensitivity, specificity for wall, lumen, and combined wall-lumen in the prediction of gangrenous cholecystitis.

\section{Results}

Table 1 shows the baseline demographics and CT values among the two groups. No significant age and gender preference was seen in the two groups. A total of $7 / 11 \mathrm{pa}-$ tients had diabetes in the gangrenous cholecystitis group. The median time interval between CT and surgery was six days in the group with gangrenous cholecystitis and 14 days in the non-gangrenous acute cholecystitis group. The unenhanced CT findings in the gallbladder for the two groups are also shown in Table 1 . The HU value of the wall was higher in the gangrenous cholecystitis group (median value of $33 \mathrm{HU}$ vs. median value of $21 \mathrm{HU}$ in the nongangrenous group; $p=0.013$ ). The HU value of lumen was higher in the gangrenous cholecystitis group (median val- 
Table 1. Patient demographics and computed tomography values in two groups

\begin{tabular}{|c|c|c|c|}
\hline Attributes & Non-gangrenous cholecystitis (nonGCh) & Gangrenous cholecystitis (GCh) & P-value \\
\hline Age, median (IQR) & $65.0(22.5)$ & $67.0(14.0)$ & $0.608^{\mathrm{a}}$ \\
\hline \multicolumn{4}{|l|}{ Gender, $n(\%)$} \\
\hline Female & $8(47.1)$ & $9(52.9)$ & \multirow[t]{2}{*}{$0.640^{b}$} \\
\hline Male & $4(66.7)$ & $2(33.3)$ & \\
\hline (T HU wall, median (range) & $21.0(8-40)$ & $33.0(18-70)$ & $0.013^{\mathrm{a}}$ \\
\hline CT HU lumen, median (range) & $8.5(1-15)$ & $21.0(8-24)$ & $<0.001^{\mathrm{a}}$ \\
\hline
\end{tabular}

${ }^{a}$ Mann-Whitney $U$ test, ${ }^{b}$ Fisher exact test, $C T$ - computed tomography, IQR - interquartile range, HU - Hounsfield units

ue of $21 \mathrm{HU}$ vs. median value of $8 \mathrm{HU}$ in the non-gangrenous group; $p<0.001$ ) (Figures 2 and 3 ). The gangrenous cholecystitis group had significantly higher $\mathrm{HU}$ values of wall and bile than the non-gangrenous group.

\section{Diagnostic accuracy of unenhanced computed tomography}

The area under the receiver operator characteristic curve for HU lumen was 0.80 (95\% CI: 0.62-0.98, $p=0.014$ ) with an ideal cut-off as $31.5 \mathrm{HU}$, where the sensitivity was $54.5 \%$ and specificity was $91.7 \%$. HU lumen has an even better assessment for gangrenous cholecystitis with AUC of its ROC as 0.92 (95\% CI: $0.80-1.00, p=0.001)$ with ideal cut-off at $12.5 \mathrm{HU}$, where the sensitivity was $81.8 \%$ and specificity was $91.7 \%$ (Figure 4 ). The combined wall-lumen cut-off is $35 \mathrm{HU}$ with sensitivity of $100 \%$ and specificity of $75 \%$ and with AUC of the ROC curve as 0.936 (95\% CI: 0.827-1.000).

\section{Discussion}

Acute cholecystitis is an acute inflammatory disease of the gallbladder, which primarily results from an ob- structive calculus impacted in the neck or cystic duct [7]. Gangrenous cholecystitis is one of the complications of acute cholecystitis, which is associated with higher morbidity and mortality rates compared to non-gangrenous cholecystitis $[8,9]$. Previous studies have described various CT findings that can predict gangrenous cholecystitis, but no single finding is specific for diagnosing this entity [4,5,10-13].

Geffroy et al. [14] and Frager et al. [15] have studied the role of unenhanced CT in diagnosing ischaemic bowel disease. Increased density in the bowel wall on unenhanced CT suggests haemorrhage and necrosis due to blockage of arterial and venous microcirculation [16]. Likewise, bacterial overgrowth with release of toxins, sepsis, suppuration, and pus is seen within the lumen of gangrenous bowel segment, which can result in a mild increase in attenuation of the intraluminal fluid contents compared to non-gangrenous bowel segment $[17,18]$. This increase in attenuation in the bowel wall and within the lumen can be expected in gangrenous cholecystitis due to its similar pathophysiology.

Our results show that the combination of unenhanced CT density value of gallbladder wall greater than $31.5 \mathrm{HU}$

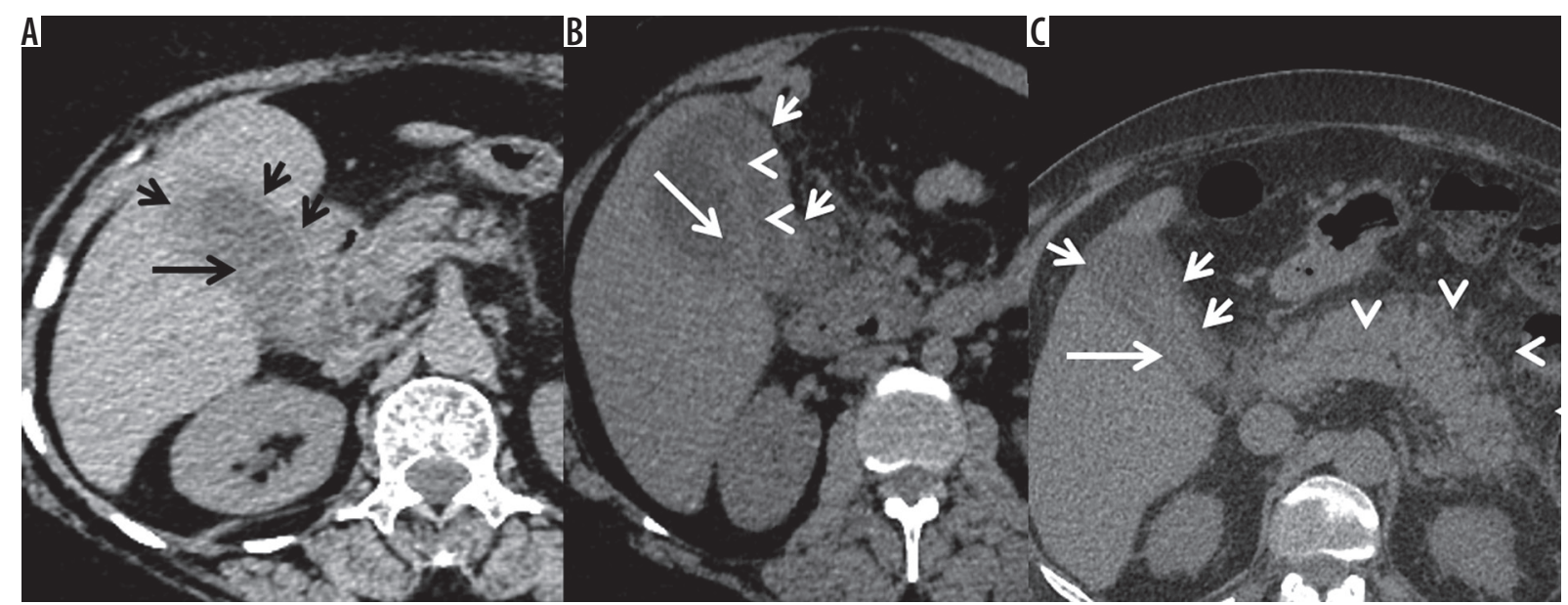

Figure 2. A) Axial non-contrast computed tomography scan of 63-year-old woman with pathologically proven gangrenous cholecystitis. Gallbladder is distended and shows high density striated wall (small arrows) and high attenuation intraluminal bile (large arrow). B) Axial non-contrast computed tomography scan of a 45 -year-old woman with pathologically proven gangrenous cholecystitis. Gallbladder is distended and shows high-density thickened wall (small arrows), high-attenuation intraluminal bile (large arrow), and intraluminal membranes (arrowheads). C) Axial non-contrast computed tomography scan of a 66-year-old man with pathologically proven acute non-gangrenous cholecystitis. Gallbladder is partially distended and shows high-density thickened wall (small arrows) and high-attenuation intraluminal bile (large arrow). Note is also made of interstitial oedematous pancreatitis (arrowheads) 

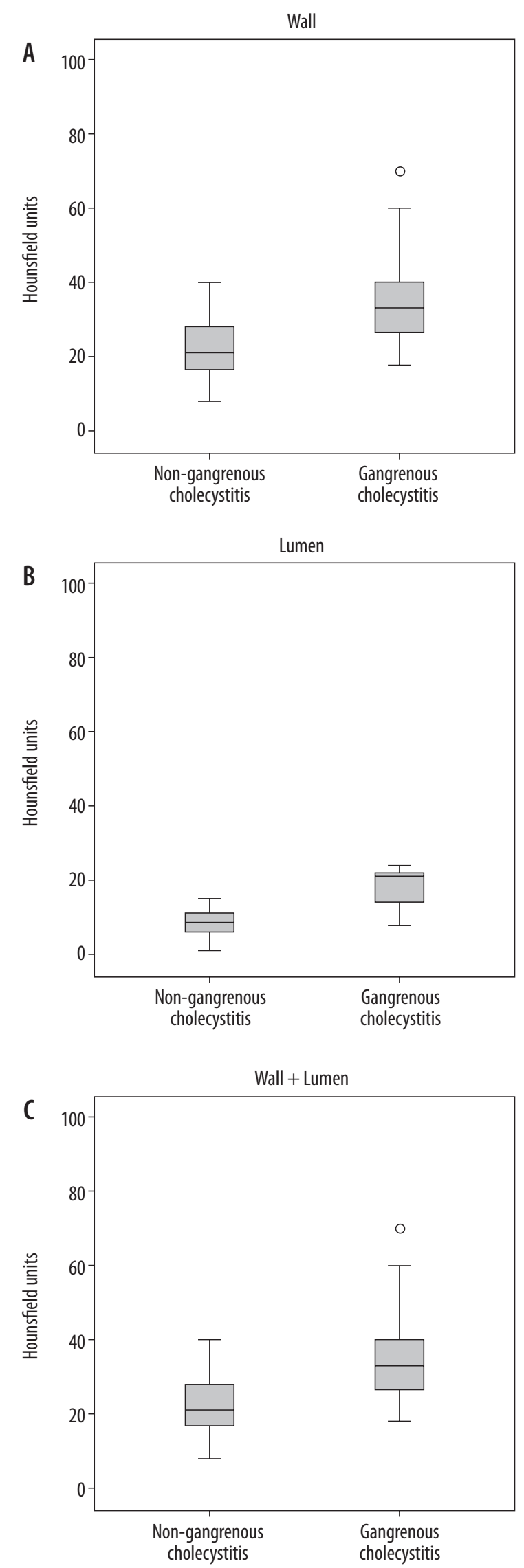

Figure 3. A) Box-and-whisker plots for the HU wall, B) HU lumen, and C) wall plus lumen of gangrenous and non-gangrenous cholecystitis. Centre horizontal line - median, bottom and top edges of box $-25^{\text {th }}$ and $75^{\text {th }}$ percentiles, vertical line - range of data

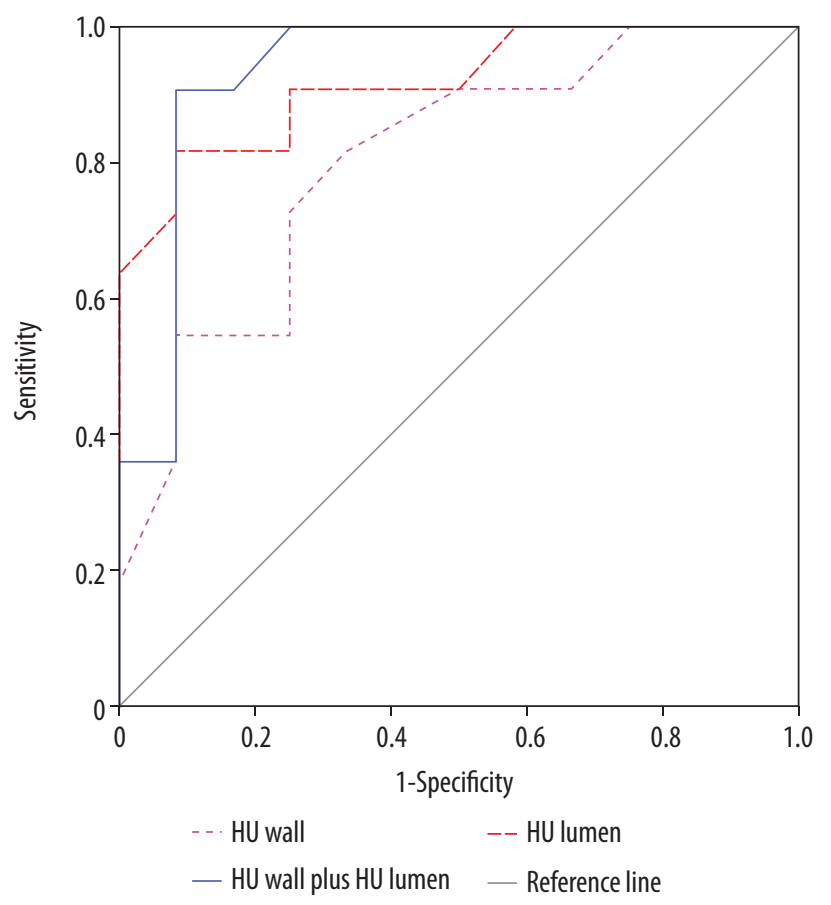

Figure 4. ROC curves showing the diagnostic performance of computed tomography in the diagnosis of combined wall-lumen sign for gangrenous cholecystitis

and intraluminal bile more than $12.5 \mathrm{HU}$ in a case of acute cholecystitis can predict gangrenous change with high sensitivity and accuracy. Interestingly, Cheng et al. [4] described hyperdense gallbladder wall sign in 2004 and noted gallbladder wall hyperattenuation (mean $40.89 \mathrm{HU}$ ) on unenhanced CT scans in $51 \%(n=27)$ of the patients in their study. However, the hyperdense wall sign was only seen in $25 \%(n=7)$ of the patients in the study done by Chang et al. [5]. Sureka et al., in their study of 31 cases on contrast-enhanced CT, concluded that the presence of more than one of these findings: gallbladder distension (short axis diameter of $\geq 4 \mathrm{~cm}$ ), intraluminal membranes, mural striation, absent or decreased enhancement of gallbladder wall suggest high probability of gangrenous change in acute cholecystitis [13]. To our knowledge, the importance of considering both gallbladder wall and intraluminal bile attenuation values on unenhanced CT scan or combined hyperdense gallbladder wall-lumen sign for diagnosing gangrenous cholecystitis has not been described before.

Our study had a few limitations, which should be highlighted and can be an area of future research. The sample size of cases of gangrenous cholecystitis was small which might have led to decrease in specificity, although there was almost 20\% increase in sensitivity. Another limitation of our study was the time lag between CT and surgical resection, which may have led to slight changes or improvement of some cases by the time of histological examination. The measured CT numbers exhibit manufacturer and $\mathrm{kVp}$ dependence, which should be taken into account when defining tolerances and cut-offs. The inter-reader agreement 
was not calculated. However, we have tried to shed light on this new CT sign 'combined hyperdense gallbladder wall-lumen sign' for diagnosing gangrenous cholecystitis.

\section{Conclusions}

In summary, our study highlights the importance of using combined hyperdense gallbladder wall-lumen sign in unenhanced CT for the diagnosis of gangrenous change in acute cholecystitis. Although the combined hyperdense gallbladder wall-lumen sign may also be present in nongangrenous cholecystitis, the cut-off CT density value of gallbladder wall greater than $31.5 \mathrm{HU}$, intraluminal bile more than $12.5 \mathrm{HU}$, and combined wall-lumen of more than $35 \mathrm{HU}$ in a known clinical setting can predict gangrenous cholecystitis with reasonable sensitivity and accuracy.

\section{Conflict of interest}

The authors report no conflict of interest.

\section{References}

1. Clifford WJ. Acute gangrenous cholecystitis. N Engl J Med 1949; 241: 640-643.

2. Weiss CA III, Lakshman TV, Schwartz RW. Current diagnosis and treatment of cholecystitis. Curr Surg 2002; 59: 51-54.

3. Revzin MV, Scoutt LM, Garner JG, Moore CL. Right upper quadrant pain: ultrasound first! J Ultrasound Med 2017; 36: 1975-1985.

4. Cheng SM, Ng SP, Shih SL. Hyperdense gallbladder wall sign: an overlooked sign of acute cholecystitis on unenhanced CT examination. Clin Imaging 2004; 28: 128-131.

5. Chang WC, Sun Y, Wu EH, et al. CT findings for detecting the presence of gangrenous ischemia in cholecystitis. AJR Am J Roentgenol 2016; 207: 302-309.

6. Marne C, Pallarés R, Martín R, Sitges-Serra A. Gangrenous cholecystitis and acute cholangitis associated with anaerobic bacteria in bile. Eur J Clin Microbiol 1986; 5: 35-39.

7. Kimura Y, Takada T, Kawarada Y, et al. Definitions, pathophysiology, and epidemiology of acute cholangitis and cholecystitis: Tokyo Guidelines. J Hepatobiliary Pancreat Surg 2007; 14: 15-26.

8. Pinto A, Reginelli A, Cagini L, et al. Accuracy of ultrasonography in the diagnosis of acute calculous cholecystitis: review of the literature. Crit Ultrasound J 2013; 5 Suppl 1: S11.

9. Fagan SP, Awad SS, Rahwan K, et al. Prognostic factors for the development of gangrenous cholecystitis. Am J Surg 2003; 186: 481-485.
10. Chawla A, Bosco JI, Lim TC, et al. Imaging of acute cholecystitis and cholecystitis-associated complications in the emergency setting. Singapore Med J 2015; 56: 438-443.

11. Shakespear JS, Shaaban AM, Rezvani M. CT findings of acute cholecystitis and its complications. AJR Am J Roentgenol 2010; 194: 1523 1529.

12. Bennett GL, Rusinek H, Lisi V, et al. CT findings in acute gangrenous cholecystitis. AJR Am J Roentgenol 2002; 178: 275-281.

13. Sureka B, Rastogi A, Mukund A, et al. Gangrenous cholecystitis: analysis of imaging findings in histopathologically confirmed cases. Indian J Radiol Imaging 2018; 28: 49-54.

14. Geffroy Y, Boulay-Coletta I, Jullès MC, et al. Increased unenhanced bowel-wall attenuation at multidetector CT is highly specific of ischemia complicating small-bowel obstruction. Radiology 2014; 270: 159-167.

15. Frager DH, Baer JW. Role of CT in evaluating patients with smallbowel obstruction. Semin Ultrasound CT MR 1995; 16: 127-140.

16. Laufman $H$, Nora PF. Physiological problems underlying intestinal strangulation obstruction. Surg Clin North Am 1962; 42: 219-229.

17. Moschetta M, Telegrafo M, Rella L, et al. Multi-detector CT features of acute intestinal ischemia and their prognostic correlations. World J Radiol 2014; 6: 130-138.

18. Barnett WO, Petro AB, Williamson JW. A current appraisal of problems with gangrenous bowel. Ann Surg 1976; 183: 653-659. 